EPJ Web of Conferences 16, 04005 (2011)

DOI: $10.1051 /$ epjconf/20111604005

(C) Owned by the authors, published by EDP Sciences, 2011

\title{
Deriving the true mass of an unresolved Brown Dwarf companion to an M-Dwarf with AO aided astrometry*
}

\author{
E. Meyer ${ }^{\mathrm{a}}$ and M. Kürster \\ Max-Planck-Institute for Astronomy (MPIA), Königstuhl 17, 69117 Heidelberg, Germany
}

\begin{abstract}
From radial velocity (RV) detections alone one does not get all orbital parameters needed to derive the true mass of a non-transiting, unresolved substellar companion to a star. Additional astrometric measurements are needed to calculate the inclination and the longitude of the ascending node. Until today only few true substellar companion masses have been determined by this method with the HST fine guidance sensor $[1,2]$. We aim to derive the true mass of a brown dwarf candidate companion to an early $\mathrm{M} 2.5 \mathrm{~V}$ dwarf with groundbased high-resolution astrometry aided by adaptive optics. We found this unique brown dwarf desert object, whose distance to the host star is only $0.42 \mathrm{AU}$, in our UVES precision RV survey of $\mathrm{M}$ dwarfs, inferring a minimum companion mass of 27 Jupiter masses [3]. Combining the data with HIPPARCOS astrometry, we found a probability of only $2.9 \%$ that the companion is stellar. We therefore observed the host star together with a reference star within a monitoring program with VLT/NACO to derive the true mass of the companion and establish its nature (brown dwarf vs. star). Simultaneous observations of a reference field in a globular cluster are performed to determine the stability of the adaptive optics (AO) plus detector system and check its suitability for such high-precision astrometric measurements over several epochs which are needed to find and analyse extrasolar planet systems.
\end{abstract}

\section{INTRODUCTION}

Radial velocity surveys to find substellar companions orbiting their host stars have led to the detection of a variety of exoplanets and brown dwarfs (BD). They also led to the definition of the "brown dwarf desert", the absence of BD companions to low-mass stars at separations $<3$ AU [4]. The BD companion frequency at separations $>1000 \mathrm{AU}$ is at least 10 times higher than that of separations of only a few $\mathrm{AU}$, which is $\approx 0.5 \%$ [5,6]. The rareness of close-in BD companions is highly significant since the commonly employed RV searches for sub-stellar companions to stars are very sensitive to such objects as the RV semi-amplitude $K$ is higher for shorter periods $P$, e.g. smaller separations from the host star. Because the RV measurements only result in a minimum companion mass and the HIPPARCOS astrometric precision is mostly not sufficient to distinguish BDs from stellar companions, the masses of the few known close-in BD candidates are often uncertain [7, 8].

We recently found a unique candidate BD companion to GJ 1046, a M2.5V star, in our UVES $\mathrm{RV}$ search for planets around $\mathrm{M}$ dwarfs [3, 9, 10]. The expected minimum astrometric signal due to the companion is 3.7 mas peak-to-peak. This value is calculated using the HIPPARCOS parallax of 71.56 mas and holds for the case that the orientation of the system is such that we only see and measure the minor axis of the orbit. This is one of the rare cases where a spectroscopic system comes into reach for astrometric observations. With astrometry the measured signal becomes stronger the more massive the companion is and the bigger the separation between the two components is. Combining the law of

*Based on observations collected at the European Southern Observatory, Paranal, Chile, programmes 381.C-0235, 281.C-5057 and 383.C-0248.

ae-mail: meyer@mpia.de

This is an Open Access article distributed under the terms of the Creative Commons Attribution-Noncommercial License 3.0, which permits unrestricted use, distribution, and reproduction in any noncommercial medium, provided the original work is properly cited. 


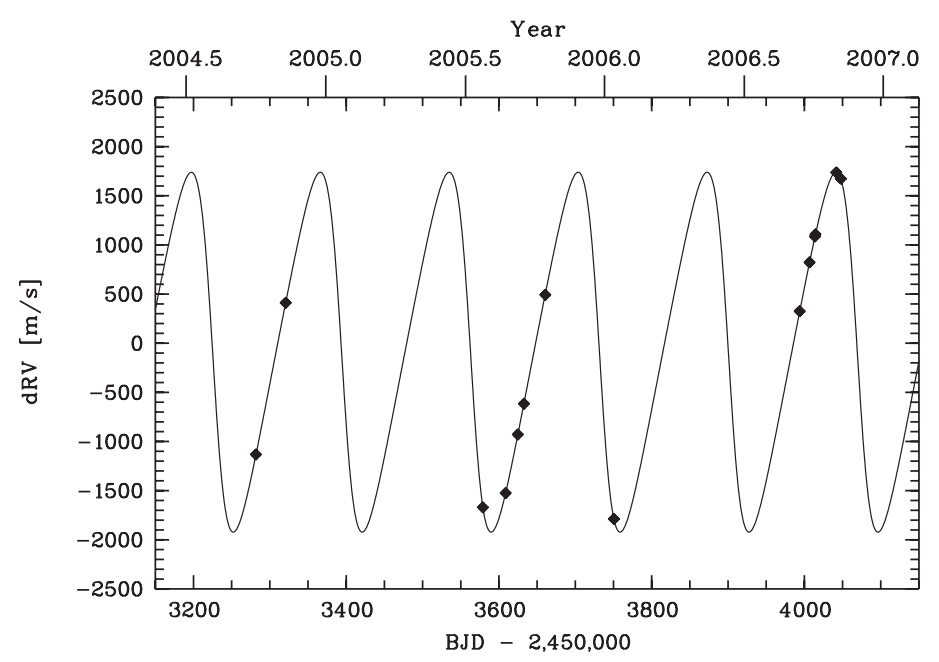

Figure 1. RV time series of GJ 1046 from [3]. The solid line corresponds to the Keplerian solution with a period of $169 \mathrm{~d}$. Note that the average measurement error is $3.63 \mathrm{~ms}^{-1}$, much smaller than the plot symbols.

lever with Kepler's $3^{\text {rd }}$ law gives the semi-amplitude $A$ of the stellar wobble in mas:

$$
A=\frac{m}{r}\left(\frac{G}{4 \pi^{2}}\right)^{1 / 3}\left(\frac{P}{M+m}\right)^{2 / 3}
$$

Here $r$ is the distance of the system [pc], $M$ is the stellar mass , $m$ the companion's mass, $P$ the orbital Period and $G$ the gravitational constant. In Figure 1 (from [3]) the time series and best-fit Keplerian orbit ( $P$ orb $=169 \mathrm{~d}, e=0.28$, semi-amplitude $K=1831 \mathrm{~ms}^{-1}$ ) of GJ 1046 is plotted. Assuming a stellar mass of $M=0.398 \mathrm{M} \odot$, a minimum companion mass $m$ min $=26.9 \mathrm{M} \mathrm{Jup}$ and an orbital semi-major axis $a=0.42 \mathrm{AU}$ was inferred. Combining the RV data with the HIPPARCOS astrometry of GJ 1046 [3] (see also [11]) a $3 \sigma$ upper limit to the companion mass of $112 \mathrm{M}$ Jup was determined. The probability for the companion to exceed the star/BD mass threshold $(0.8 \mathrm{M} \odot)$ is just $2.9 \%$.

\section{OBSERVATIONS}

We observed GJ 1046 with the adaptive optics system NACO at the VLT-UT4 within a monitoring program. For astrometric measurements a reference star, preferably close to the observed star is needed. By chance GJ 1046 is located at $\sim 30^{\prime \prime}$ separation from a suitable reference star, see Fig. 2. GJ 1046 has a magnitude of $\mathrm{V}=11.61$ and $\mathrm{K}=7.03$, the reference star has $\mathrm{V}=14.33$ and $\mathrm{K}=13.52$. The observations are made with a narrow-band filter centered on $2.12 \mu \mathrm{m}$ to minimize the effects of chromatic aberration. To derive subpixel accuracy for the astrometry one needs to know the pixelscale and the accuracy of the orientation of the detector very well. For this purpose we observed a reference field in 47 Tuc each time immediately before the science field. This field contains three bright stars and some fainter ones suitable to check the image scale and the reproducibility of the field derotation. The reference field is also needed for PSF calibration, since in case the companion is stellar, its light contribution may affect the astrometric positions, given its maximum separation by almost 0.5 FWHM from the primary. 

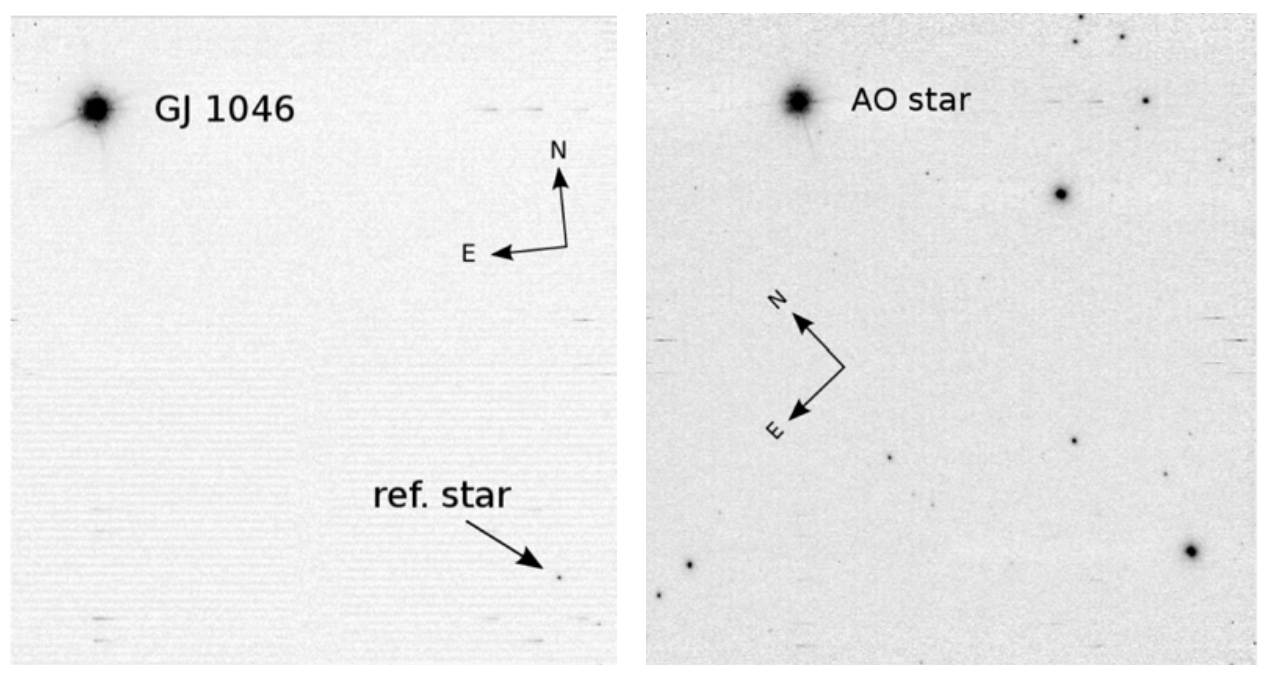

Figure 2. NACO image of GJ 1046 (left) and the reference field in the globular cluster 47 Tucanae (right) from July 2008. GJ 1046 is the bright star in the upper left corner, the reference star is located in the lower right corner. Also visible in the frames are ghosts produced by the bright star (elongated features).

\subsection{Data reduction}

We did not use the NACO-pipeline products but reduced the data on our own. The reduction was the same for the science and for the reference field. Dark, flat and badpixel corrections were carried out and the frames obtained at the same jitter positions were added. After that, we co-added the frames from the different jitter positions by using the jitter-routine [12] contained in the eclipse package. The positions of the stars were measured by fitting a Moffat-function to the individual stars using the MPFIT package [13]. To estimate the positional error resulting from the fit we resampled the PSF 100 times with the bootstrap method [14] and fitted each one again with a Moffat-function. The standard deviations of these fits from the mean position in $\mathrm{x}$ and $\mathrm{y}$ are used as estimates for the errors. Special care was taken to correct for differential atmospheric refraction and differential aberration. The final images after coaddition of the single frames are shown in Figure 2.

\subsection{Astrometric orbit determination}

The astrometric signal derived from the data is a combination of the stellar parallax, proper motion and the signal due to the companion. To extract the orbital motion of the star one needs to account for the parallax and proper motion carefully. Figure 3, left panel, shows an example of a simulated astrometric signal of GJ 1046 with a combination of parallax, proper motion and orbital motion. The parameters are: $P=168.848 \mathrm{~d}, T 0=4651.5 \mathrm{~d}(\mathrm{BJD}-2,450,000), e=0.2793, a=1.940 \mathrm{mas}, \omega=92.70^{\circ}$ as derived from the RV measurements, $\mu \alpha=1394.10 \mathrm{mas} / \mathrm{yr}, \mu \delta=550.05 \mathrm{mas} / \mathrm{yr}, \pi=71.56 \mathrm{mas}$ from the HIPPARCOS data and $i=45^{\circ}, \mathrm{X}=60^{\circ}$ assumed. As the proper motion of GJ 1046 is very high, 1497 mas/yr, it totally dominates its motion and pulls the parallax + orbital ellipse apart into a wave-like motion.

If we subtract the proper motion in our simulations, one can see the orbital motion of the star due to its companion, still dominated by the parallax (Fig. 3, middle panel). The curve is not a perfect ellipse as in the case of pure parallax motion and is not closing after one full orbit of the Earth around the sun. Finally in the right panel of Fig. 3 the pure orbital motion without parallax and proper motion is displayed. As one can see, it is very important to know/measure the parallax, to distinguish the orbital 

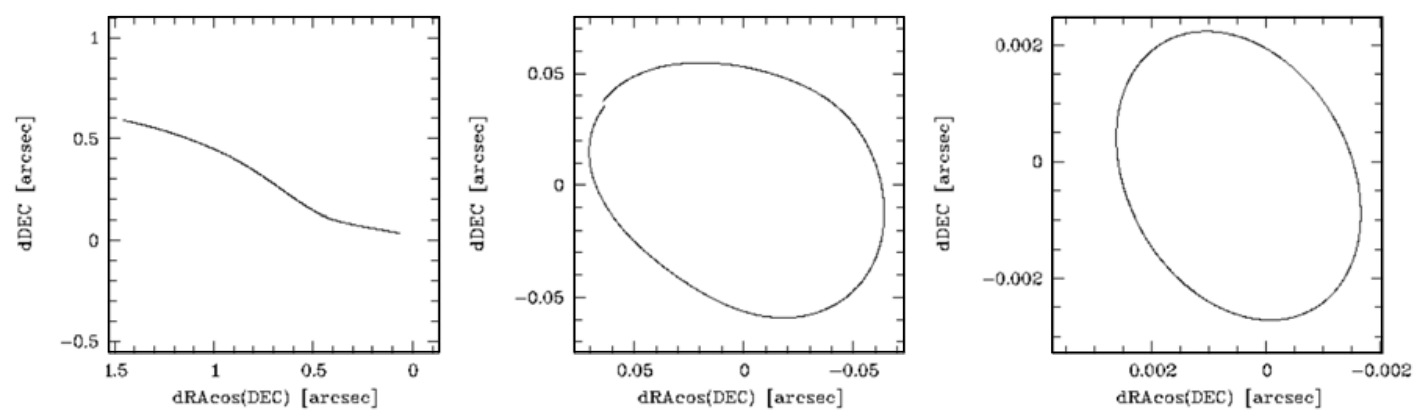

Figure 3. Change in declination versus right ascension. The left panel shows a simulated astrometric signal for GJ 1046 (see text for details). The other two panels show the simulated astrometric signal without proper motion and with (middle) and without (right) parallax, respectively.

motion from it. Note the different scales in the changes of right ascention and declination in the three different cases.

Using the Hipparcos parallax (71.56 mas) we predict the minimum astrometric signal (full minor axis) of the stellar reflex motion to be 3.7 mas peak-to-peak corresponding to 0.136 image pixels (px) in the CONICA S27 camera, but with the true effect possibly much greater. For an object at the BD-star border $(0.8 \mathrm{M} \odot)$ the full minor axis would extend 11.5 mas or $0.42 \mathrm{px}$. At the Hipparcos derived upper mass limit it would be 15.4 mas or $0.57 \mathrm{px}$.

\subsection{Plate-scale and derotation stability}

We observed every run right before our science target a reference field in the globular cluster 47 Tucanae (see Figure 2). These observations are used to determine the plate-scale changes and the derotation stability between the different epochs. Therefore the positions of the stars are measured and corrected for differential aberration and differential atmospheric refraction in the same way as the science frames. We then calculated the shift in $x / y$, the scale-changes and the rotation of the single epochs to the mean epoch in time. First results show a maximum plate-scale variation of $0.2 \%$ and a derotator instability of $\approx 0.6 \mathrm{deg}$ between two epochs. These first results show, that in multi-epoch astrometry the most challenging task is to measure and correct such effects very precisely to obtain mas or even microarcsec astrometric solutions.

\section{CONCLUSION}

From radial velocity observations one can only derive a minimum mass for substellar companions. Combined with astrometric follow-up observations one can derive the true companion mass. We are currently investigating a promising brown dwarf companion to an early M type star with NACO to prove its $\mathrm{BD}$ nature. The data is obtained and the data reduction is done. We are currently investigating the stability of the plate-scale and the rotation of the detector over several epochs. The positional precision measured in the single epochs without taking any errors from plate-scale changes or derotation error into account, are promising so that an astrometric detection of the companion is possible and likely.

We want to thank the ESO OPC for the time allocation and the Paranal Science Operations Team for carrying out the service mode observations. 
Research, Science and Technology of Brown Dwarfs and Exoplanets

\section{References}

[1] G. F. Benedict, et al., ApJ, (2002), 581, L115-L118

[2] G. F. Benedict, et al., AJ, (2006), 132, 2206-2218

[3] M. Kürster, M. Endl, S. Reffert, $A \&$ \& A, (2008), 483, 869-874

[4] G. W. Marcy, R. P. Butler, PASP, (2000), 112, 137-140

[5] J. E. Gizis, J. D. Kirkpatrick, A. Burgasser, et al., ApJ, (2001), 551, L163

[6] R. Neuhäuser, E. W. Guenther, $A \& A$, (2004), 420, 647

[7] D. Pourbaix, $A \& A,(2001), 369$, L22

[8] D. Pourbaix, F. Arenou, $A \& A$, (2001), 372, 935

[9] M. Kürster, M. Endl, F. Rouesnel, et al., $A \&$ \& A, 2003, 403, 1077

[10] M. Zechmeister, M. Kürster, M. Endl, ArXiv e-prints, (2009), 0908.0944

[11] S. Reffert, A. Quirrenbach, $A \&$ \& , (2006), 449, 699

[12] N. Devillard, ASPC, (1999), 172, 333

[13] C. B. Markwardt, ASPC, (2009), 411, 251

[14] B. Efron, Anuals of Statistics, (1979), 7(1), 1-2-6 